INVESTIGACIONES

\title{
RECURSOS SEMIOTICOS DEL PROFESOR DE MATEMATICA: FUNCIONES COMPLEMENTARIAS DEL HABLA Y LOS GESTOS PARA LA ALFABETIZACION CIENTIFICA ESCOLAR
}

\author{
SEMIOTIC RESOURCES OF TEACHERS OF MATHEMATICS: COMPLEMENTARY FUNCTIONS \\ OF DISCOURSE AND GESTURES FOR SCIENTIFIC LITERACY AT SCHOOLS
}

\author{
Recursos semióticos do professor de matemática: funções complementares \\ da fala e os gestos para a alfabetização científica escolar
}

\section{Dominique Manghi Haquin}

Pontificia Universidad Católica de Valparaíso, Fono: 2721109

dmanghi@gmail.com, Valparaíso, Chile

\begin{abstract}
RESUMEN
Los profesores de matemática despliegan un discurso pedagógico mediante diversos recursos semióticos, condicionados por la dinámica escolar prototípica del aula escolar: la interacción cara a cara y el uso del pizarrón. Esta investigación busca describir, desde la perspectiva multimodal de la comunicación, las funciones de los principales recursos semióticos utilizados por los docentes para la regulación del conocimiento disciplinar. Para esto se llevó a cabo un estudio de casos múltiples constituido por dos profesores de $1^{\text {er }}$ año de Enseñanza Media y sus clases de matemática. Los resultados nos indican que los recursos semióticos principales son el habla y los gestos deícticos, los cuales se despliegan en configuraciones de significado particulares con objetivos determinados. Habla y gestos construyen de manera conjunta o intersemiótica el conocimiento matemático, y constituyen una combinación especializada propia de la pedagogía para la alfabetización científica.
\end{abstract}

Palabras clave: multimodalidad, alfabetización científica, discurso pedagógico, semiosis.

\begin{abstract}
Teachers of mathematics unfold their pedagogical discourse through a range of semiotic resources, under the conditions of prototypical classroom dynamics that is face to face interaction and the use of the whiteboard. From a multimodal perspective, this research seeks to describe the main functions of the semiotic resources used by teachers for the regulation of disciplinary knowledge. A multiple case study was developed based on the performance of two teachers of freshmen and their lessons of mathematics. The results show that the main semiotic resources are speech and deictic gestures, which unfold in meaning configurations with specific purposes. Speech and gestures together, or in an inter-semiotical way build the mathematical knowledge and make up for a specialized combination typical to the pedagogy of scientific literacy.
\end{abstract}

Key words: multimodality, scientific literacy, pedagogical discourse, semiosis.

\section{RESUMO}

Os professores da matemática realizam um discurso pedagógico mediante diversos recursos semióticos, condicionados pela dinâmica escolar prototípica da sala de aula escolar: a interação cara a cara e o uso do quadro negro.Esta investigação procura descrever, desde a perspectiva da multi-modalidade da comunicação, as funções dos principais recursos semióticos utilizados pelos professores para a regulação do conhecimento disciplinar. Para isto, levou-se a cabo um estudo de casos múltiplos constituído por dois professores de $1^{\circ}$ ano de Ensino Secundário e suas aulas de matemática. Os resultados nos indicam que os recursos semióticos principais são a fala e os gestos, os quais se realizam em configurações de significado particulares com objetivos determinados. A fala e os gestos constroem o conhecimento matemático de maneira conjunta ou intersemiótica, e constituem uma combinação especializada própria da pedagogia para a alfabetização científica.

Palavras-chave: multimodalidade, alfabetização científica, discurso pedagógico, semiosis. 


\section{INTRODUCCION}

Los estudios acerca del discurso del profesor y su rol para la alfabetización escolar tradicionalmente han asumido dos creencias. La primera es que el lenguaje constituye el medio principal y único para enseñar y aprender en la escuela, mientras que la segunda creencia asume que hay sólo una forma de usar el lenguaje en el colegio, y que esa forma sirve para comunicarse y aprender de manera eficiente en todas las asignaturas escolares.

Estos supuestos largamente aceptados por los estudiosos de la lengua han tenido importantes repercusiones en la pedagogía de la alfabetización, ya que presentan un panorama comunicativo simplificado y ubican en un lugar preponderante a la lengua materna, como el medio exclusivo y autónomamente eficiente para la comunicación, el aprendizaje y la enseñanza dentro del ámbito educativo (Kress \& van Leeuwen, 2001; Kress, 2001; Jewitt, 2008).

Como respuesta a estos supuestos, la Lingüística Sistémica Funcional y la perspectiva multimodal de la comunicación nos brindan otra posible manera de mirar las aulas escolares, la cual enriquece el paisaje comunicativo y la manera de comprender los contextos en los que ponemos en juego los recursos para crear significados. El cambio de énfasis ha repercutido en los estudios sobre alfabetización y ha dado paso a dos ideas acerca del aprendizaje de la comunicación en la escuela. La primera se relaciona con las formas de representación y comunicación del conocimiento y su relación con las comunidades que las crean y utilizan. Los profesores y sus discursos pedagógicos recontextualizan los discursos de contextos primarios, como por ejemplo de los contextos científicos, en los contextos escolares. Esto implica que los docentes reproducen en el aula aquellas maneras especiales de representar y comunicar dichos conocimientos específicos (Bernstein, 1990). Las investigaciones acerca del conocimiento científico y su discurso en el ámbito escolar se han vuelto centrales para estudiar el aprendizaje en las diferentes disciplinas escolares, enfatizando en las particularidades de cada comunidad disciplinar y sus formas especiales de representación de su cúmulo de conocimientos (Maton, 2007).

La segunda idea tiene que ver con la ampliación del foco de los recursos para la comunicación hacia la mirada multimodal del paisaje comunicativo. En la actualidad las investigaciones acerca de la alfabetización específica a las disciplinas ya no sólo se concentran en los discursos construidos mediante el lenguaje escrito, sino que consideran que el conocimiento se construye en base a múltiples recursos semióticos como gráficos, mapas, imágenes, entre otros (Unsworth, 2001; Martin, 2009; O’Halloran, 2007). Del mismo modo, para su enseñanza el profesor pone en juego recursos multimodales para crear significado y regular el conocimiento de los aprendices (Hood, 2009).

La presente investigación explora desde la perspectiva multimodal el discurso pedagógico en la interacción cara a cara del aula de matemáticas de $1^{\text {er }}$ año de Enseñanza Media, para describir algunos de los recursos del profesor especialista en pedagogía de la matemática para la enseñanza de esta disciplina. Para esto revisaremos los fundamentos teóricos de la alfabetización científica escolar entendida desde la perspectiva multimodal y sus etapas. Luego presentaremos la metodología y los resultados respecto de la pregunta de investigación planteada: ¿Qué rol cumplen habla y gestos como recursos semióticos en la enseñanza de las matemáticas de profesores especialistas? 


\section{FUNDAMENTACION TEORICA}

\section{ALFABETIZACION COMO COMUNICACION MULTIMODAL}

Desde las teorías de la Lingüística Sistémica Funcional (en adelante LSF) y de la perspectiva multimodal, el aprendizaje de la representación y comunicación es parte de un proceso amplio y complejo, referido a múltiples formas de significar y comunicar situadas y legitimadas social y culturalmente (Lemke, 1990). Tal como nos propone la semiótica social, dichas formas de crear significado han sido moldeadas en el tiempo por los diversos grupos sociales, entre ellos los que constituyen comunidades disciplinares, por ejemplo biología, medicina o matemática. Ellos han seleccionado una combinación de recursos semióticos para cubrir las necesidades de representación y comunicación de sus integrantes y sus funciones sociales y culturales (Hodge \& Kress, 1988; Kress, 2001; van Leeuwen, 2000; Kress \& van Leeuwen, 2001).

En los últimos años se ha desarrollado, principalmente en los países anglosajones, la preocupación por lo que denominan multiliteracies (alfabetizaciones múltiples). Este término da cuenta de la ampliación del foco de la enseñanza del dominio del lenguaje escrito hacia el reconocimiento de otros recursos semióticos involucrados en la representación y comunicación de las ideas de una comunidad, así como también de la inclusión de los recursos tecnológicos para el aprendizaje en el aula, los cuales abren posibilidades complejas y específicas a estos medios y sus formas de comunicar (New London Group, 2000). Este reconocimiento de la complejidad y variedad de los recursos para significar debiera además tener consecuencias en la formación de profesores y en el diseño curricular de los años escolares (Christie, 2005; Martin, 2007).

Destacaremos dos repercusiones producto de esta mirada compleja a la comunicación disciplinar en el aula. Una primera consecuencia importante es que el panorama semiótico al interior de la escuela nos revela diferentes recursos para crear significado que se ponen en juego en la actividad escolar. La variedad de medios semióticos va desde la interacción cara a cara y el uso de la pizarra prototípicos de la sala de clase, hasta medios tecnológicos como presentaciones proyectadas desde el computador. De la misma manera, el rango de modos o recursos semióticos utilizados en el aula se amplía desde la consideración del lenguaje oral y escrito hacia la inclusión de dibujos, fotos, mapas, tablas, gráficos, entre otros (Kress, Ogborn, \& Martins, 1998). En el caso de las clases de matemáticas, los medios prototípicos utilizados por los profesores son la interacción cara a cara, el pizarrón, el cuaderno, la guía escrita y la prueba escrita (Manghi, 2009). Estos se ponen en juego de manera integrada mediante el simbolismo matemático, las imágenes -como gráficos y tablas- y la escritura, constituyendo estos tres los recursos semióticos centrales que construyen el conocimiento matemático (O’Halloran, 2005). Una de las dificultades reconocidas del aprendizaje del discurso matemático es que en su despliegue presenta sucesivas traducciones de un código a otro (Duval, 2000). Por ejemplo, un conocimiento que puede estar representado lingüísticamente luego se representa en un gráfico o en simbolismo matemático. Estas traducciones son denominadas por O'Halloran (2005) como macrotransiciones semióticas y son foco del quehacer didáctico de los docentes especialistas en pedagogía de las matemáticas (Manghi, 2009).

Una segunda repercusión relevante tiene que ver con reconocer que el proceso de alfabetización que recorren los escolares en su vida educativa no es un trayecto homogéneo. 
A medida que los niños van avanzando en sus años de escolarización, deben involucrarse en formas de significar y tareas de alfabetización más avanzadas, en las cuales la lengua y los otros recursos para significar se usan de manera cada vez más lejana a la interacción informal cotidiana (Schleppegrell, 2004) o al discurso horizontal (Bernstein, 1990). A partir de esta idea, podemos identificar ciertas etapas críticas en la vida escolar de un estudiante, desde su recorrido por la educación parvularia, general básica, media hasta la enseñanza superior. En cada una de ellas el alumno se ve enfrentado a cambios y períodos de transición entre una etapa escolar y la siguiente, cada una con sus objetivos y rutinas de actividades, organización curricular, dinámica y participantes diferentes, asî como formas de representar y comunicar particulares (Christie, 2005).

Esta perspectiva para aproximarse al problema de la alfabetización pone de relieve que para aprender la disciplina es necesario acceder a las formas gramaticales de los recursos semióticos utilizados para representarlos. Es decir, no sólo es necesario aprender los conocimientos de cada disciplina sino también la forma valorada de representarlos y comunicarlos. Los contenidos y sus discursos constituyen un único proceso integral que construye el conocimiento particular de cada disciplina (Unsworth, 2001). A continuación nos detendremos a describir la evolución de la enseñanza de la alfabetización.

ENSEÑANDO A COMUNICARSE EN CADA ETAPA ESCOLAR: HACIA LA ALFABETIZACION CIENTIFICA

La LSF ha recogido la definición de discurso pedagógico desde la sociología de la educación (Bernstein, 1990) para describir las características de los discursos, que circulan y se intercambian en las salas de clases, mayormente anglosajonas, en las diversas disciplinas escolares y niveles educativos (Haliday \& Martin,1993; van Leeuwen \& Humphrey, 1996; Coffin, 1997; Martin \& Veel, 1998; Unsworth, 2001; Schleppegrell, 2004; Christie, 2002; Oteíza, 2006). Las descripciones textuales de la LSF son relevantes en la medida en que permiten dar cuenta de la ontogénesis del dominio de los recursos semióticos de los niños y adolescentes. Debido al reconocimiento de estas etapas críticas y transiciones, los investigadores proponen que es necesario enriquecer el modelo de alfabetización considerando la naturaleza de los recursos para significar.

A partir de esta propuesta, podemos observar de otra manera lo que ocurre en la escuela. Durante la educación parvularia, los niños se aproximan desde sus diversos orígenes a unas formas/funciones especiales de crear significado en la escuela para interactuar en el aula y en el espacio escolar. Aquí la comunicación comienza a diferenciarse del discurso cotidiano familiar y son las educadoras de párvulos las encargadas de llevar a cabo este acercamiento. De acuerdo a la investigación de Rail (2007) en aulas chilenas, la reformulación del discurso de la ciencia en esta etapa escolar es llevada a cabo por las educadoras, principalmente mediante definiciones lingüísticas de diverso tipo, las cuales se distancian excesivamente del discurso científico original. El repertorio de estrategias de definición, todas lingüísticas orales, nos revela que a estos pequeños aprendices se les muestra el mundo científico como un mundo de objetos y no de acciones, lo que reinterpretado desde la descripción del discurso científico corresponde a un conocimiento fuera del sentido común (Martin, 1993). Este estudio revela además la ausencia de contenido propiamente científico en el discurso pedagógico de las educadoras, así como de las maneras apropiadas de representarlo y comunicarlo, lo que delata la escasa formación científica en las educadoras de párvulos (Rail, 2007). 
$\mathrm{Al}$ entrar a enseñanza básica y en especial en los primeros años escolares, los esfuerzos educativos se concentran explícitamente sobre la enseñanza/aprendizaje de una forma dominante de significar: el código escrito. La enseñanza de los contenidos escolares y de su comunicación es llevada a cabo en esencia por profesores de enseñanza general básica, cuya labor es hacerse cargo de la instrucción de los variados sectores de aprendizaje curricular mediante los recursos semióticos disponibles en la práctica escolar. Al mismo tiempo y de una forma menos explícita, enseñan acerca de la valoración de ciertas maneras de utilizar tanto el lenguaje oral como el escrito y otros sistemas semióticos considerados eficientes para el aprendizaje escolar general (Veel, 1997, No citado 1998; Unsworth, 2008). En sus prácticas pedagógicas ponen en juego diversos medios típicos del aula escolar utilizados de forma especial en enseñanza básica. Entre dichos medios semióticos ocupa un lugar importante el texto o manual escolar (Rail 2007; Meneses 2006). Estos presentan significados construidos mediante una combinación de recursos semióticos visuales, no sólo la escritura. Los aprendices son introducidos progresivamente a fotos, dibujos, íconos, mapas, esquemas, etc. (Pereira, 2009).

Según la investigación de Meneses (2008), en quinto año básico, el texto o manual de estudio es el centro del quehacer pedagógico. Los profesores creen que una vez que los estudiantes aprenden a codificar y decodificar la lengua escrita, pueden comenzar a utilizarla automáticamente para el aprendizaje desde los textos escolares de las diversas disciplinas. La lectura y la escritura están presentes principalmente como un medio de acceso y registro de información en todas las áreas curriculares en estudio.

Destaca en este estudio la homogeneidad de las actividades educativas programadas, realizadas y evaluadas por los profesores de enseñanza básica en torno al uso central del texto escolar. Las distinciones entre los diferentes subsectores emergen de la propuesta de las actividades diseñadas en los mismos textos escolares. En el subsector de Lenguaje se encuentran sobre todo actividades de análisis y comunicación de información mediante el lenguaje escrito. El subsector de Comprensión de la Sociedad se caracteriza por la combinación de tareas de adquisición y análisis de información lingüística junto con la de información gráfica proveniente de los mapas. En cuanto a Comprensión de la Naturaleza, los textos presentan tareas didácticas en que se utilizan la lectura y la escritura para el análisis de la información, principalmente en situaciones de experimentación. Finalmente, en el subsector de Matemática, las tareas poseen objetivos de interpretación de la información, en las que se mezcla el código verbal con el código simbólico y numérico (Meneses, 2008).

Al llegar a la enseñanza media o secundaria, y en algunos establecimientos en los últimos años de la enseñanza básica, los encargados de enseñar a los estudiantes pasan a ser los profesores especialistas formados en sus respectivas disciplinas para la enseñanza de cada subsector de aprendizaje: matemáticas, biología, historia, física, etc. En esta etapa, los alumnos deben aprender a pertenecer simultáneamente a diversas asignaturas, cada una de ellas corresponde a un subsector de aprendizaje curricular que es nutrido por una disciplina y/o ciencia y posee sus formas especiales de comunicación y representación.

Los estudios acerca de la alfabetización en enseñanza secundaria en países anglosajones nos indican que se debe considerar la naturaleza de los diferentes contenidos escolares, su alfabetización específica y tecnicismos (Martin, 1999; Unsworth, 2001), ya que las diferencias en los recursos lingüísticos y semióticos utilizados en los diversos discursos disciplinares se relacionan con diferencias epistemológicas, así como metodológicas y pedagógicas (Schleppegrell, 2004). 
En esta etapa, los textos escolares son utilizados con mayor énfasis en Historia y Geografía (Mineduc, 2006), siendo menos usados en el área de matemática. Cada profesor especialista requiere lograr que las formas de crear significado de sus aprendices se acerquen a una configuración semiótica esperada, donde el habla y la escritura juegan un rol, junto con los otros recursos en un diseño semiótico peculiar para su disciplina como esquemas, fórmulas, manipulación de objetos, diagramas, mapas, etc. (Veel, 1997). Dicha manera particular de crear significado no siempre es enseñada en forma visible y explícita como un contenido curricular más, sino que más bien es aprendida en la interacción áulica, como un tipo de conocimiento tácito del experto que debe ser apropiado por los aprendices recién llegados, moldeando la identidad o la conciencia de los aprendices (Christie, 2002). Así, el profesor especialista y sus recursos para regular el aprendizaje de la disciplina en esta última etapa escolar juegan un rol fundamental en la alfabetización disciplinar y científica.

\section{METODOLOGIA}

El objetivo de este estudio apunta a describir habla y gestos como recursos que utiliza el profesor especialista en matemáticas para la enseñanza de los conocimientos y del discurso de su disciplina. Con este fin, observamos a dos profesores de matemáticas en interacción con su grupo de estudiantes para analizar, desde la perspectiva multimodal, los discursos y recursos semióticos que ponían en juego.

Para lograr el objetivo propuesto, la aproximación a nuestro objeto de estudio se llevó a cabo desde el paradigma cualitativo e involucró dos enfoques teórico-metodológicos distintos aunque relacionados. El enfoque de la semiótica social (Jewitt \& Owama, 2001; Martin \& Rose, 2008) se utilizó para recolectar los datos y el análisis multimodal del discurso de la vertiente de la semiótica social (Kress, 2001; Jewitt, 2008; Martin, 2009) para explorarlos.

El diseño metodológico corresponde a un estudio de casos múltiples de tipo cualitativo, en el que cada caso se define como único. Además, los casos se consideran instrumentales, ya que no interesan como caso particular en sí, sino como representantes de un fenómeno (Ruiz, 2003). Esta investigación analiza el discurso pedagógico a partir de dos casos correspondientes a dos profesores de matemática y sus clases frente al grupo de estudiantes de $1^{\text {er }}$ año de Enseñanza Media. La selección de los dos colegios particulares subvencionados de la V Región se llevó a cabo de manera intencionada, considerando aquellos bien evaluados en la Prueba SIMCE de $2^{\circ}$ año Medio. Otros dos criterios fueron tomados en cuenta. Por una parte, interesó observar profesores formados en pedagogía de la matemática, para describir la manera en que regulan el conocimiento de los estudiantes. Por otra parte, nos enfocamos en el $1^{\text {er }}$ año de Enseñanza Media porque, en la mayoría de los casos, corresponde al nivel escolar en que los estudiantes entran en contacto con los profesores especialistas, iniciando una nueva etapa en su trayectoria escolar y en el proceso de alfabetización.

En cuanto al procedimiento de recolección del material, este consideró dos condiciones. La primera se refiere a la naturaleza del objeto de estudio: discursos multimodales y dinámicos. Los discursos de los profesores se consideran como textos complejos debido a que son construidos mediante múltiples recursos semióticos, es decir, de manera 
multimodal. Además, la naturaleza dinámica o construcción contingente del discurso, propio de la interacción cara a cara, en contraposición con textos estáticos como los impresos, es central a la decisión respecto de la recolección de material. La segunda condición se relaciona con el enfoque metodológico que considera los contextos y comunidades en que se producen e interpretan dichos textos. Esto implica que el material debía registrar las diversas acciones semióticas de los profesores en la interacción cara a cara con su grupo de estudiantes. Por este motivo, el registro de la interacción áulica es de tipo audiovisual, y se llevó a cabo mediante dos cámaras en el aula (una estática y una dinámica), para poder observar la totalidad del panorama semiótico. Finalmente, cabe señalar que se grabó la misma unidad pedagógica completa en los dos estudios de caso, la que corresponde a la unidad "las proporciones".

El material registrado fue analizado desde dos focos. Primero cada profesor y su discurso pedagógico como casos únicos, y luego considerando sus textos como un tipo de texto prototípico de la clase de matemática, para observar los patrones de significado a un nivel más general en busca de rasgos comunes.

En cuanto al análisis de los datos, utilizando el análisis del discurso multimodal de vertiente de la semiótica social, se desarrollaron dos grandes fases: la fase de análisis macro o satelital y la fase de análisis micro o textual.

a) Análisis macro o satelital del discurso (Kress, Ogborn \& Martins, 1998): este es un acercamiento al material grabado desde el nivel del contexto, observando los patrones de significado como patrones de comportamiento en el aula. Este nivel permite segmentar el texto en unidades con sentido.

b) Análisis micro o textual del discurso: este es un acercamiento al material seleccionado desde el análisis del texto multimodal dinámico. Martin (2009) propone la exploración metafuncional de los significados construidos de manera multimodal. En nuestro caso, nos hemos enfocado en el análisis ideacional, esto es, en la representación de quién hace qué, a quién, cuándo, dónde, por qué y cómo (Eggins \& Martin, 2003).

De esta manera, una vez realizado el análisis satelital y la segmentación del material grabado en patrones de significado, se llevó a cabo un análisis micro que contempló el análisis ideacional (Martin \& Rose, 2006), a partir de las trascripciones multimodales de los microgéneros principales de una sesión de clases prototípica en cada caso. Este nivel permitió describir con detalle los roles de los recursos semióticos en la enseñanza de la matemática.

\section{RESULTADOS}

En primer lugar, los resultados del análisis multimodal del discurso del profesor nos indican que los profesores en interacción con el grupo de estudiantes ponen en juego tres recursos semióticos principales: habla o lenguaje oral, gestos y simbolismo matemático y, además, otros dos recursos de manera secundaria: escritura e imágenes dibujadas. Todos ellos van en función del uso del pizarrón como medio protagonista de la enseñanza de las proporciones, ya que la interacción cara a cara también está condicionada por el uso del pizarrón. Los gestos no son de cualquier tipo, sino que destacan principalmente los gestos para la deixis o indicación (Mc Neil, 2000), es decir, los gestos deícticos. 
En segundo lugar, cabe destacar que estos recursos no son utilizados por los profesores de manera azarosa. Por una parte, dichos recursos semióticos son desplegados por los docentes de manera sistemática, en ciertos momentos de la sesión de clases y para determinados objetivos. Por otra parte, estos recursos son puestos en juego por los profesores de manera combinada. Ambos profesores de matemática utilizan principalmente las combinaciones semióticas habla y gestos deícticos, así como también habla y simbolismo matemático.

Respecto del uso sistemático de los recursos semióticos esto es posible de observar en los patrones de significado del análisis satelital. Tal como observamos en el ejemplo del cuadro 1, podemos encontrar una configuración de significados común a ambos profesores: el análisis de razonamiento (AR), la que es realizada de manera íntegra por la combinación habla-gestos deícticos. En este caso, el patrón de significado o género es muy frecuente en la etapa intermedia de la unidad pedagógica. Los docentes utilizan este género con el objetivo de comentar para los aprendices los procedimientos desarrollados previamente en el pizarrón. La combinación habla-gestos deícticos permite los comentarios del docente respecto del procedimiento de la disciplina en todas las etapas del género.

\section{Cuadro 1}

Patrón de significados para comentar, combinación habla-gestos

\begin{tabular}{|c|c|c|c|c|c|}
\hline $\begin{array}{l}\text { Etapas } \\
\text { AR }\end{array}$ & $\begin{array}{l}\text { 1. Anuncio } \\
\text { de categoría de } \\
\text { procedimiento }\end{array}$ & $\begin{array}{l}\text { 2. Descripción } \\
\text { de la condición }\end{array}$ & $\begin{array}{l}\text { 3. Indicación } \\
\text { de opciones de } \\
\text { procedimiento }\end{array}$ & $\begin{array}{l}\text { 4. Descripción } \\
\text { de la condición }\end{array}$ & $\begin{array}{l}\text { 5. Indicación } \\
\text { de opciones de } \\
\text { procedimiento }\end{array}$ \\
\hline $\begin{array}{l}\text { Acciones } \\
\text { semióticas }\end{array}$ & & & & & \\
\hline Medios & Cara a cara & $\begin{array}{l}\text { Cara a cara } \\
\text { (Pizarra) }\end{array}$ & $\begin{array}{l}\text { Cara a cara } \\
\text { (Pizarra) }\end{array}$ & $\begin{array}{l}\text { Cara a cara } \\
\text { (Pizarra) }\end{array}$ & $\begin{array}{l}\text { Cara a cara } \\
\text { (Pizarra) }\end{array}$ \\
\hline $\begin{array}{l}\text { Modos o } \\
\text { recursos } \\
\text { semióticos }\end{array}$ & $\begin{array}{l}\text { Lenguaje oral } \\
\text { y gestos }\end{array}$ & $\begin{array}{l}\text { Lenguaje oral } \\
\text { y gestos }\end{array}$ & $\begin{array}{l}\text { Lenguaje oral } \\
\text { y gestos }\end{array}$ & $\begin{array}{l}\text { Lenguaje oral y } \\
\text { gestos }\end{array}$ & $\begin{array}{l}\text { Lenguaje oral } \\
\text { y gestos }\end{array}$ \\
\hline
\end{tabular}

Esta misma función es posible de observar en otro género o patrón de significados recurrente en la etapa intermedia de la unidad pedagógica estudiada: el modelamiento de procedimiento (MP). En este otro caso, en la mayoría de las etapas genéricas predomina la combinación habla-simbolismo matemático cuyo objetivo es ejemplificar. Sin embargo, en este género hay una etapa particular en la que ambos profesores detienen el flujo de dicha combinación para comentar, utilizando nuevamente para esto habla y gestos de manera entrelazada. Esto puede ser observado en el cuadro 2, etapa $\mathrm{N}^{\mathrm{o}} 3$. 


\section{Cuadro 2}

Patrón de significados para ejemplificar, etapa 3:combinación habla-gestos

\begin{tabular}{|l|l|l|l|l|l|}
\hline $\begin{array}{l}\text { Etapas } \\
\text { MP }\end{array}$ & $\begin{array}{l}\text { 1. Descripción } \\
\text { del mundo } \\
\text { matemático }\end{array}$ & $\begin{array}{l}\text { 2. Trascripción } \\
\text { simbólica }\end{array}$ & $\begin{array}{l}\text { 3. Escritura } \\
\text { notacional }\end{array}$ & $\begin{array}{l}\text { 4. Aplicación } \\
\text { de operaciones }\end{array}$ & $\begin{array}{l}\text { 5. Respuesta a } \\
\text { la pregunta }\end{array}$ \\
\hline $\begin{array}{l}\text { Acciones } \\
\text { semióticas }\end{array}$ & & & & &
\end{tabular}

Debido a la relevancia de la combinación habla-gestos deícticos, el análisis micro profundiza en sus funciones en el discurso pedagógico de los docentes y su rol en la regulación del conocimiento de los aprendices. El análisis multimodal de estos géneros y etapas genéricas nos señala que los profesores utilizan ambos recursos de manera complementaria entre sí para representar el conocimiento disciplinar.

Consideraremos los gestos como un sistema semiótico que se pone en juego junto con otros recursos corporales como la postura, la mirada, la expresión facial, es decir, los gestos como una acción corporeizada (Hood, 2009). La mayoría de los gestos identificados en este estudio corresponden al tipo deíctico, esto es, cumplen la función de indicar elementos del contexto, en este caso, elementos del simbolismo en la pizarra. Mientras que el profesor mediante el habla despliega un texto, simultáneamente mediante los gestos indica elementos simbólicos de la pizarra, representándolos como objetos materiales presentes en la sala de clases. Esto implica que el uso semiótico de los gestos no construye un texto gestual propiamente tal, no obstante, los elementos indicados por los gestos activan su significado y son entrelazados en el texto lingüístico convirtiéndolo en textos multimodales que combinan habla, gestos deícticos y simbolismo matemático, este último como elemento indicado. La coutilización de gestos junto con el habla permite establecer un repertorio de relaciones intersemióticas que funcionan como opciones para significar en las clases de matemática.

El uso complementario de ambos recursos semióticos se basa en los potenciales de cada uno de ellos para crear significado. El lenguaje ya ha sido descrito como una herramienta humana para la clasificación o tipologización (Lemke, 1990). Esto significa que el lenguaje ha evolucionado para cumplir la función de representar y comunicar tipologías o taxonomías y, por lo tanto, pensar y actuar en torno a ellas. La tipologización es algo propio del discurso de la ciencia (Halliday \& Martin, 1993). En este discurso científico, en nuestro caso el matemático, primero el profesor define lingüísticamente elementos representándolos mediante relaciones de identificación ("la proporción es una relación 
entre razones"). Luego de representarlos como una definición, incluye el elemento definido como parte de una taxonomía (proporciones directas, proporciones indirectas, por ejemplo). El lenguaje nos posibilita esta construcción de un mundo abstracto en el que los elementos se entienden de manera técnica.

En nuestros estudios de caso, los docentes de matemática utilizan el habla como recurso semiótico para construir un metadiscurso matemático (O’Halloran, 2005). Esto se lleva a cabo en coordinación con el uso de gestos deícticos. Tal como mencionamos anteriormente, la coutilización de ambos recursos por parte de los docentes ocurre en todo el patrón de significados que hemos denominado análisis de razonamiento (AR) y en la tercera etapa del patrón modelamiento de procedimiento (MP). Mediante el habla, los profesores definen para los alumnos la manera en que las entidades deben ser entendidas en el mundo de la ciencia (Martin \& Rose, 2008) y esto lo llevan a cabo a través de las definiciones y taxonomías, tanto lingüísticas como simbólicas matemáticas. La pregunta que surge frente a dicha situación pedagógica es cómo es que los docentes enseñan estas definiciones y taxonomías técnicas. De qué manera regulan el conocimiento matemático de sus aprendices que recién se inician en esta etapa, en que el profesor especialista es quien imparte la clase de matemática.

Los hallazgos de esta investigación nos indican que no sólo el habla del docente cumple un rol didáctico para la enseñanza del conocimiento disciplinar, sino que junto con ella aparecen los gestos deícticos que modifican y enriquecen las definiciones y taxonomías matemáticas. De esta manera, cuando el profesor representa una tipología de conocimiento disciplinar mediante el habla, el gesto deíctico que emplea simultáneamente agrega otro tipo de significado dirigido a sus estudiantes. Esto es posible de observar en los ejemplos 1 y 2 relacionados con el potencial semiótico de ambos recursos y el rasgo espacial del simbolismo matemático, mientras que los ejemplos 3 y 4 se refieren a la construcción de taxonomías en el conocimiento matemático por parte de los docentes.

El primer ejemplo lo encontramos cada vez que un profesor define simultáneamente una relación entre elementos del mundo matemático utilizando los dos recursos. En este caso, el habla representa una relación técnica entre elementos ("80 es a Ana como 5 es a 3”) y los gestos indican esta misma relación simbólica que ya ha sido escrita en el pizarrón, tal como se observa en el cuadro 3.

\section{Cuadro 3}

Definición simultánea, habla-gestos deícticos

\begin{tabular}{|l|c|c|}
\hline \multirow{2}{*}{ Definición simultánea } & Gesto deíctico & \\
& & \\
\cline { 2 - 3 } & Habla & 80 es a Ana como 5 es a 3 \\
\hline
\end{tabular}


Esto nos muestra que la definición matemática puede ser representada tanto por el habla como por el simbolismo matemático; sin embargo, el habla del profesor no incluye la descripción topológica de cada elemento de la definición simbólica en el pizarrón. El gesto deíctico del docente pone de relieve las características topológicas del simbolismo matemático que no se representan mediante el habla. Es decir, el gesto muestra la definición espacial y simbólica matemática de la proporción, información que no está incluida en la definición lingüística de la misma. Dicha disposición espacial de los elementos simbólicos de una proporción también parece ser objeto de enseñanza por los docentes. Aunque los docentes no expresan lingüísticamente la importancia de aprender cómo ubicar cada elemento en la pizarra para construir el significado matemático, los gestos deícticos que usan parecen indicarnos que la traducción desde la definición lingüística -originalmente en el informe lingüístico del problema de proporciones- hacia la definición simbólica matemática es un aspecto didáctico en el que ponen atención. Para apoyar la traducción entre estos dos recursos semióticos, ambos docentes recurren a la combinación habla-gesto deíctico.

El segundo caso en que encontramos habla y gesto deíctico puestos en juego por los profesores de manera simultánea es en la representación de circunstancias. El habla de los profesores en el comentario de los procedimientos es dependiente del simbolismo matemático escrito previamente en el pizarrón. De esta manera, los docentes comentan lingüísticamente utilizando circunstancias de lugar y de modo de manera ambigua (así, acá, ver cuadro 4), desambiguándolas mediante el uso de los gestos deícticos. Los profesores utilizan los gestos para especificar tanto la ubicación espacial en puntos determinados del procedimiento simbólico en la pizarra, así como maneras de representar las operaciones matemáticas, es decir, apuntando a colocaciones simbólicas matemáticas como igualdades, razones o proporciones, todas ellas construidas mediante diversos elementos simbólicos que se distribuyen en el espacio de manera convencional. Esto da cuenta del potencial del simbolismo matemático coordinado con los gestos deícticos, ambos en el canal visual, los que pueden especificar disposiciones espaciales de mejor manera que el lenguaje. Este potencial es utilizado por ambos profesores para enfatizar el carácter topológico del simbolismo matemático.

\section{Cuadro 4}

Relación ambigüedad/especificación habla-gestos

\begin{tabular}{|l|l|l|l|}
\hline Gesto deíctico & $\begin{array}{l}\text { Circunstancia } \\
\text { específica }\end{array}$ & $\begin{array}{l}\text { la única diferencia que al } \\
\text { pasarla acá }\end{array}$ & ipor qué multiplicaron así? \\
\hline Habla & ambigua
\end{tabular}


Por lo tanto, en el ejemplo 1 así como en el 2, el gesto deíctico resulta fundamental para andamiar la equivalencia en esta traducción desde lenguaje oral al simbolismo matemático, este último siempre con una distribución espacial particular a las proporciones, representación espacial que también porta significado. El potencial del simbolismo matemático y el gesto deíctico sobre él tiene que ver con las características del canal visual.

Dicha definición simultánea funciona como una sinonimia intersemiótica (Royce, 2007), no obstante, no es plenamente equivalente, ya que apela siempre a una expansión de significado (Lemke, 1990) dependiente del potencial semiótico de cada recurso involucrado en la representación. En este caso, el potencial del canal visual aporta significado topológico (Lemke, 1990) y el simbolismo matemático ha evolucionado para aprovechar este potencial para el lenguaje matemático.

Respecto del tercer ejemplo en el cual habla y gestos funcionan de manera coordinada, este corresponde a los casos en que el profesor utiliza el habla para representar una clase o tipología y los gestos deícticos para señalar miembros simbólicos pertenecientes a ese tipo. En estos casos, el profesor utiliza el habla para establecer una categoría abstracta y simultáneamente, mediante los gestos deícticos dinámicos, delimita una zona espacial del pizarrón donde se encuentran los miembros de esa categoría o clase (ver cuadro 5). Esta manera de coutilizar los recursos semióticos llevada a cabo por los profesores colabora en la construcción de una taxonomía del mundo de la matemática en diferentes niveles de abstracción. Con el habla los docentes construyen las taxonomías matemáticas, mientras que con los gestos dinámicos sobre el simbolismo matemático en la pizarra entrelazan dichas categorías a un conjunto de elementos miembros de aquella categoría.

\section{Cuadro 5}

Relación clase/miembro habla-gestos

\begin{tabular}{|l|l|l|l|}
\hline Gesto deíctico & Miembros de una clase & & \\
\hline Habla & clase & los kilómetros cuadrados & los habitantes \\
\hline
\end{tabular}

La última coutilización de habla y gestos deícticos de los profesores en estos estudios de casos, también se refiere a la construcción de tipologías de manera intersemiótica. En estos casos, el docente usa el habla para construir categorías abstractas (por ejemplo: antecedente más consecuente) y los gestos señalan ejemplares simbólicos de la pizarra para cada tipo (por ejemplo, Ana + Julia), u otros ejemplos como los del cuadro 6. Esta relación utilizada por los profesores cumple una función didáctica distinta a las anteriores, ya que el lenguaje aporta aquí la tecnicalización construyendo taxonomías disciplinares, mientras que los gestos deícticos indican ejemplares para cada tipo abstracto. 


\section{Cuadro 6}

Relación tipo/ejemplar lenguaje oral y gestos

\begin{tabular}{|l|l|l|l|}
\hline Gesto deíctico & ejemplar & & \\
\hline Habla & tipo & la variable, & una razón \\
\hline
\end{tabular}

Los ejemplos 3 y 4 nos muestran cómo ambos profesores utilizan el potencial semiótico de los dos recursos para enriquecer la construcción de taxonomías para sus aprendices: el lenguaje y su potencial tipológico, así como los gestos deícticos y su potencial material. De esta manera, mientras que con el lenguaje construyen elementos técnicos, al mismo tiempo con los gestos deícticos muestran elementos simbólicos representativos de cada categoría, escritos previamente en la pizarra. Con esta manera de construir las taxonomías matemáticas, los dos profesores logran jugar con los niveles de abstracción, en pos de favorecer la apropiación de los estudiantes de estas categorías técnicas.

De esta manera, podemos reconocer en ambos estudios de caso ciertos elementos que son parte de la representación multimodal y que constituyen conocimiento matemático que deben aprender los estudiantes. El primer elemento corresponde a la disposición espacial de los símbolos, es decir, los estudiantes deben aprender a manejar el potencial topológico del simbolismo matemático. Ambos profesores se preocupan de mostrar la disposición espacial de los elementos a los aprendices, tanto la disposición entre ellos -como por ejemplo en las colocaciones que conforman una proporción-, así como la disposición en el despliegue en el pizarrón, esto es, en el desarrollo de los ejercicios a medida que se llevan a cabo las traducciones o macrotransiciones intersemióticas. El segundo elemento corresponde a las taxonomías matemáticas propias del tema de las proporciones. Los dos docentes utilizan las funciones complementarias del habla y gestos deícticos para mostrar las categorías de la taxonomía en dos niveles de abstracción al mismo tiempo.

\section{CONCLUSIONES}

Los hallazgos de esta investigación nos revelan que los profesores combinan habla y gestos deícticos para construir los significados de manera entrelazada. La perspectiva multimodal evidencia que el lenguaje no carga con todo el significado, ya que su potencial semiótico permite ciertas funciones pero otras no. Los profesores realizan la representación del conocimiento disciplinar a través del uso de ambos recursos semióticos de manera integrada, con el objetivo de comentar para los aprendices los procedimientos matemáticos de la pizarra. Es decir, el significado creado por los docentes estaría incompleto si es representado utilizando exclusivamente el habla o sólo los gestos. El significado completo se logra por la combinación de recursos, los que cumplen funciones complementarias tanto 
para la representación de la disposición espacial del simbolismo como para la construcción de las taxonomías técnicas en diferentes niveles de abstracción. Esta construcción del significado entre dos o más recursos semióticos de manera coordinada es lo que los investigadores denominan intersemiosis (O'Halloran, 2005).

La combinación habla-gestos deícticos para la enseñanza de la matemática es un recurso común en ambos docentes estudiados. La coutilización de ellos podría ser parte de la tradición de la pedagogía de la matemática, es decir, una combinación de recursos que ha evolucionado para servir a los propósitos comunicativos de la comunidad de profesores de matemática.

Los resultados de esta investigación nos llevan a reflexionar acerca de la importancia del medio cara a cara, en coordinación con el uso del pizarrón, para la pedagogía de alfabetización científica llevada a cabo por profesores especialistas en matemática. Un profesor especialista que sólo utilice el modo lingüístico para enseñar matemática sin apoyarse en el pizarrón representa exclusivamente las tipologías, presentando el conocimiento a los aprendices como un saber abstracto y general, alejado de la experiencia de los estudiantes. En cambio, un profesor que utilice simultáneamente ambos recursos: habla y gestos deícticos, por una parte, construye integralmente tanto el conocimiento tipológico matemático así como el conocimiento topológico del simbolismo y, por otra, representa el conocimiento abstracto o alejado del sentido común y al mismo tiempo muestra ejemplares de las categorías escritas en el pizarrón, acercándolo al mundo material de la sala de clases más cercano a la experiencia de los estudiantes. Es decir, la interacción cara a cara junto con el uso del pizarrón favorece que los docentes aprovechen los potenciales semióticos del habla y los gestos deícticos y, más precisamente, los potenciales semióticos de la combinación de ambos, con fines didácticos.

La educación moderna apela a un supuesto trasversal a las diferentes etapas escolares. Dicho supuesto plantea que existiría alguna forma de continuidad entre la especialización de la identidad de los aprendices en la trayectoria educativa. Esto implica que el desempeño escolar en matemática de alguna manera predice el desempeño universitario en matemática y este repercute finalmente en su desempeño eficiente en el ámbito matemático en la vida adulta (Muller, 2006). Mirando este continuo de más cerca, distinguimos en él diferentes etapas en la trayectoria escolar que nos indican que la dirección general del desarrollo de la alfabetización disciplinar de los aprendices se distancia progresivamente de la experiencia de sentido común propia de la niñez, hacia la construcción de la experiencia alejada de lo inmediato, volviéndose cada vez más abstracta y fuera del sentido común (Berntein, 1990; Martin, 1993). El desplazamiento hacia la expresión de la experiencia fuera del sentido común se ve facilitado por el dominio cada vez mayor de la gramática no sólo de la escritura sino que también de los demás recursos semióticos (Christie, 2005). Podríamos agregar a estas etapas del trayecto escolar, la influencia de profesores cada vez más especializados en la disciplina que enseñan.

Esta última idea pone de relieve no sólo las etapas educativas dentro del supuesto continuo sino que además la existencia de transiciones entre ellas. ¿Cuáles son las diferencias entre la pedagogía de la alfabetización científica entre una etapa y la siguiente? ¿Qué rol juegan los profesores como mediadores de dichas transiciones? Los hallazgos de esta investigación nos indican que los docentes especialistas utilizan ciertas combinaciones semióticas para facilitar esta inmersión de los estudiantes en la especificidad 
disciplinaria de la Enseñanza Media. Sin embargo, queda mucho por conocer respecto de esta transición y las otras. La pedagogía de la alfabetización científica debería considerar los desafíos de la ontogénesis del aprendizaje escolar del lenguaje y otros recursos semióticos, para lograr la participación efectiva en la Enseñanza Media y vida adulta de los aprendices.

\section{BIBLIOGRAFIA}

Bernstein, B. (1990). Class, codes and control vol. 4: The structuring of pedagogic discourse. Londres: Routledge.

Bernstein, B. (2000). Pedagogy, Symbolic Control and Identity. Londres: Rowan \& Littlefield.

Christie, F. (2002). Classroom Discourse Analysis: A functional perspective. Londres: Continuum.

Christie, F. (2005). Advanced literacy development for the years of adolescence. En Darwin Symposium Series Imagining Childhood - Children, Culture and Community, 1-17.

Coffin, C. (1997). Constructing and giving value to the past: An investigation into Secondary School History. En F. Christie \& J. Martin (Eds.), Genre and institutions: Social processes in the work place and school (pp. 196-230). Londres: Continuum.

Duval, R. (2000). Basic issues for research in mathematics education. En T. Nakahara \& M. Koyama (Eds.), Proceedings of the 24th Conference of the International Group for the Psychology of Mathematics Education, vol. 1 (pp. 55-69). Hiroshima: Hiroshima University.

Eggins, S. \& Martin, J. (2003). El contexto como género: una perspectiva lingüística funciona. En Revista Signos, 36 (54), 185-205.

Halliday, M.A.K. \& Martin, J. (1993). Writing science: Literacy and discursive power. Londres: Falmer Press.

Hodge, B. and Kress, G. (1988). Social Semiotics. Cambridge: Polity.

Hood, S. (2009). Embodying language. En S. Drefus, S. Hood \& S. Stenglin (Eds.), Semiotic Margins. Meaning in multimodalities (pp. 34-48). Londres: Equinox.

Jewitt, C. (2008). Multimodal classroom research. En AERA Review of Research in Education, 32, 241-267.

Jewitt, C \& R Owama (2001). Visual Meaning: a social semiotic approach. En T. van Leeuwen \& C. Jewitt (Eds.), Handbook of Visual Analysis (pp. 134-156). Londres: Sage.

Kress, G. (2001). Multimodality [en línea]. Disponible en: http://www.ched.uct.ac.za/literacy/ Papers/KressPaper.html.

Kress, G., Ogborn, J., \& Martins, I. (1998). A satellite view of language. En Language Awareness, $2 \& 3,69-89$.

Kress, G. \& van Leeuwen, T. (2001). Multimodal Discourse. The Modes and Media of Contemporary Communication. Londres: Arnold.

Lemke, J. (1990). Talking Science. Language, learning and values. Nueva Jersey: Ablex.

Manghi, D. (2009). Coutilización de recursos semióticos para la regulación del conocimiento disciplinar. Multimodalidad e intersemiosis en el Discurso Pedagógico de Matemática en $1^{\text {er }}$ año de Enseñanza Media. Tesis para optar al grado de doctor en lingüística. Valparaíso: Pontificia Universidad Católica de Valparaíso.

Martin, J. (1993). Genre and literacy - modelling context in educational linguistics. En Annual Review of Applied Linguistics, 13, 141-172.

Martin, J. (1999). Mentoring semogenesis: "genre-based" literacy pedagogy. En Christie, F. (Ed.), Pedagogy and the shaping of consciousness. Linguistic and social processes. Londres: Continuum. 
Martin, J. (2009). Boomer dreaming: the texture of re-colonisation in a lifestyle magazine. En G. Forey \& G. Thompson (Eds.), Text type and texture (pp. 260-182). Londres: Equinox.

Martin, J. (2007). Construing knowledge: a functional linguistic perspective. En F. Christie \& J. Martin (Eds.), Language, knowledge and pedagogy (pp. 34-64). Londres: Continuum.

Martin, J. \& Rose, D. (2006). Working with Discourse: Meaning beyond the clause. Londres: Continuum.

Martin, J. \& Veel, R. (1998). Reading science. Critical and functional perspectives on dicourses of science. Londres: Routledge.

Martin, J. \& Rose, D. (2008). Genre relations: mapping culture. Londres: Equinox.

Maton, K. (2007). Knowledge-knower structures in intellectual and educational fields. En F. Christie \& J. Martin (Eds.), Language, knowledge and pedagogy: functional linguistics and sociological perspective (pp. 87-108). Londres: Continuum.

McNeill, D. (Ed.) (2000). Language and gesture: window into thought and action. Cambridge: Cambridge University Press.

Meneses, A. (2006). Leer y escribir en la escuela: representaciones discursivas de los diferentes agentes educativos en las áreas prioritarias del currículo escolar en NB3. Entre contenidos máximos y objetivos funcionales. Tesis para optar al grado de doctor en lingüística. Valparaíso: Pontificia Universidad Católica de Valparaíso.

Mineduc (2006). Estudio sobre uso de textos escolares en Enseñanza Media. [En línea]. Disponible en: http://portal.textosescolares.cl/website/index2.php?id_seccion=5\&id_contenido=4

Muller, J. (2006). On the shoulders of giants: verticality of knowledge and the school curriculum. En R. Moore, M. Arnot, J. Beck \& H. Daniels (Eds.), Knowledge, power and educational reform. Nueva York: Routledge.

New London Group, The (2000). A pedagogy of multiliteracies: Designing social futures. In B. Cope \& M. Kalantzis (Eds.), Multiliteracies: Literacy learning and the design of social futures (pp. 9-38). London: Routledge.

O'Halloran, K. (2005). Mathematical Discourse: Language, Visual Images and Symbolism. Londres: Continuum.

O'Halloran, K. (2007). Mathematical and Scientific Forms of Knowledge: A Systemic Functional Multimodal Grammatical Approach. En F. Christie \& J. Martin (Eds.), Language, Knowledge and Pedagogy: Functional Linguistic and Sociological Perspective (pp. 205-36). Londres: Continuum.

Oteíza, T. (2006). Discurso pedagógico de la historia. Un análisis lingüístico sobre construcción ideológica de la historia de Chile (1970-2001). Santiago: Frasis.

Pereira, F. (2009). El rol de las imágenes en los textos escolares de lenguaje y comunicación. Ponencia del Congreso SOCHIL. Santiago, Chile.

Rail, S. (2007). Estrategias de reformulación utilizadas por los profesores en los discursos de divulgación científica en contextos de educación infantil. Tesis para optar al grado de doctor en lingüística. Valparaíso: Pontificia Universidad Católica de Valparaíso.

Royce, T. (2007). Intersemiotic complementarity: a framework for multimodal discourse analysis. En T. Royce \& W. Bowcher (Eds.), New directions in the analysis of the multimodal discourse (pp. 63-109). Nueva Jersey: Lawrence Erlbaum Associates.

Ruiz, J. (2003). Metodología de la investigación cualitativa. Bilbao: Universidad de Deusto.

Schleppegrell, M. (2004). The language of schooling. A Functional Linguistics Perspective. Nueva Jersey: Lawrence Erlbaum Associates.

Unsworth, L. (2001). Teaching Multiliteracies across the Curriculum: changing contexts of text and image in classroom practice. Buckingham: Open University Press.

Unsworth, L (Ed.). (2008). Multimodal Semiotics and Multiliteracies Education: Transdisciplinary approaches to research and professional practice. Londres: Continuum. 
van Leeuwen, T \& Humphrey, S. (1996). On learning to look through a geographer's eyes. En R. Hasan \& R. Williams (Eds.), Literacy in Society (pp. 29-49). Londres: Longman.

Veel, R. (1997). Learning how to mean- scientifically speaking: apprenticeship into scientific discourse in the secondary school. En F. Christie \& J. Martin (Eds.), Genre and Institutions: social processes in the workplace and school (pp. 160- 194). Londres: Painter. 
